\title{
Palawaniaceae fam. nov., a new family (Dothideomycetes, Ascomycota) to accommodate Palawania species and their evolutionary time estimates
}

\section{Mapook $A^{1,2,3}$, Hyde KD ${ }^{1,2,3}$, Hongsanan $S^{3}$, Phukhamsakda $C^{1,3}$, Li $J^{1,2,3}$, Boonmee $\mathbf{S}^{3^{*}}$}

${ }^{1}$ Key Laboratory for Plant Diversity and Biogeography of East Asia, Kunming Institute of Botany, Chinese Academy of Sciences, Kunming 650201, Yunnan, People's Republic of China

${ }^{2}$ World Agroforestry Centre, East and Central Asia, Kunming 650201, Yunnan, People's Republic of China

${ }^{3}$ Center of Excellence in Fungal Research, Mae Fah Luang University, Chiang Rai 57100, Thailand

Mapook A, Hyde KD, Hongsanan S, Phukhamsakda C, Li JF, Boonmee S 2016 - Palawaniaceae fam. nov., a new family (Dothideomycetes, Ascomycota) to accommodate Palawania species and their evolutionary time estimates. Mycosphere 7 (11), 1732-1745, Doi 10.5943/mycosphere/7/11/8

\begin{abstract}
Palawania species are common on palms, occurring on dried fronds and spines, rarely on leaves, at first appearing as solitary, circular, black spots and then being confluent and lacking superficial hyphae. The taxonomy of the genus has been problematic because of lack of some important morphological characters as well as molecular data. Two collections made in Thailand are characterized based on analyses of combined LSU, SSU and RPB2 sequence datasets. Phylogenetic analyses indicate that Palawania species form a sister clade with Pleurotremataceae (= Dyfrolomycetaceae) and have a close relationship with Muyocopronales and Acrospermales. Thus, we introduce a new family Palawaniaceae (Dothideomycetes family, incertae sedis). A new Palawania species is also introduced from northern Thailand based on its distinct phylogeny and comparison of morphological characteristics. The present study clarifies the phylogenetic placement of Palawania and divergence time estimates are provided for the new family.
\end{abstract}

Key words - Ascomycota - Dothideomycetes - evolution - new species - phylogeny - taxonomy

\section{Introduction}

The genus Palawania was introduced by Sydow \& Sydow (1914) and is typified by Palawania grandis (Niessl) Syd. \& P. Syd. The genus was previously place in the family Microthyriaceae (Müller \& von Arx 1962, Lumbsch \& Huhndorf 2010, Wu et al. 2011, Hyde et al. 2013, Wijayawardene et al. 2014) based on its morphological characteristics being similar to Microthyrium, while sharing some characters with Asterinaceae (Wu et al. 2011, Hongsanan et al. 2014). Index Fungorum (2016) list seven species epithets for the genus. However, five species (i.e. P. brosimi Bat. \& J.L. Bezerra, P. dovyalidis (Doidge) G.C. Nel, P. eucleae (Doidge) G.C. Nel, P. halleriae (Dippen.) Doidge, P. orbiculata (Syd. \& P. Syd.) Doidge, have been transferred to other genera in the family Parmulariaceae. Two species (P. cocois Syd. \& P. Syd and P. grandis (Niessl) Syd. \& P. Syd) from palms were accepted in the family Microthyriaceae. Material of P. grandis (Niessl) Syd. \& P. Syd. was re-examined by Wu et al. (2011) who provided descriptions, together 
with illustrations. However, fresh collections and sequence data are needed to confirm the relationship and phylogenetic placement of Palawania (Wu et al. 2011).

The aim of the present study is to determine the phylogenetic placement of the genus Palawania. Divergence time estimates for Palawaniaceae are provided. A new species is also introduced, based on molecular and morphological comparison with descriptions, together with illustrations.

\section{Materials \& Methods}

\section{Sample collection and specimen examination}

Fresh materials were collected from Chiang Rai Horticultural Research Center during 2013-2014. Fungal micromorphology was studied following the methods of Mapook et al. (2016a). Single spore isolations were obtained using the methods of Chomnunti et al. (2014). Ascospores germinating within 12-24 h were transferred to new malt extract agar (MEA) plates and incubated at $25-30{ }^{\circ} \mathrm{C}$ in the dark, one of these cultures was used for molecular study. The specimens and living cultures are deposited in the Herbarium of Mae Fah Luang University (Herb. MFLU) and Culture collection Mae Fah Luang University (MFLUCC), Chiang Rai, Thailand and Herbarium of Cryptogams, Kunming Institute of Botany Academia Sinica (HKAS), China. Facesoffungi numbers and Index Fungorum numbers were registered as outlined in Jayasiri et al. (2015) and Index Fungorum (2016).

\section{DNA extraction, PCR amplification and sequencing}

Isolates were grown on MEA at $25-30{ }^{\circ} \mathrm{C}$ for two weeks. The fungal mycelium was scraped off and transferred to microcentrifuge tubes $(1.5 \mathrm{ml}$.). The fungal genomic DNA from mycelium was extracted by the Biospin Fungus Genomic DNA Extraction Kit (BioFlux ${ }^{\circledR}$, China) following the manufacturer's instructions (Hangzhou, P.R. China). The fungal genomic DNA was extracted directly from the ascomata using The E.Z.N.A. ${ }^{\circledR}$ Forensic DNA Kit (Omega Bio-Tek, Inc., United States) following the manufacturer's protocol. DNA amplifications were performed by polymerase chain reaction (PCR). The partial large subunit nuclear rDNA (LSU) was amplified with primer pairs LROR and LR5 (Vilgalys \& Hester 1990). The partial small subunit nuclear rDNA (SSU) was amplified with primer pairs NS1 and NS4 (White et al. 1990). The partial RNA polymerase second largest subunit (RPB2) was amplified by using primers fRPB2-5F and fRPB27cR (Liu et al. 1999). Methods for PCR amplification and sequencing were performed as in Mapook et al. (2016a).

\section{Phylogenetic analysis}

The most closely related taxa were determined using nucleotide BLAST searches online in GenBank (http://www.ncbi.nlm.nih.gov/). Combined LSU, SSU and RPB2 sequence data from representative closest relatives to Palawaniaceae were selected following Hongsanan et al. (2016) and Mapook et al. (2016b), to confirm the phylogenetic placement of the Palawania strains. Representative stains from Lecanoromycetes were selected as outgroup taxa based on their placement close to the ingroup, following Hongsanan et al. (2016). The closest matched taxa were determined through nucleotide blast searches in GenBank. The sequences used for analyses with accession numbers are given in Table 1. All sequence data were aligned using MAFFT (v7.110) online program (http://mafft.cbrc.jp/alignment/server/) (Katoh \& Standley 2013). The alignments were checked and uninformative gaps minimized manually where necessary in BioEdit 7.0.1 (Hall 1999). Maximum likelihood (ML) and Bayesian inference (BI) analyses were used in the following the methodology as described in Mapook et al. (2016a). The GTR+I+G model with inverse gamma rate were selected for each partition based on the results from MrModeltest v. 2.2 (Nylander et al. 2004). Phylogenetic trees were drawn using Treeview v. 1.6.6 (Page 1996). The new nucleotide sequence data are deposited in GenBank. 


\section{Fossil calibrations}

The fossil calibrations were used in the analyses following the methodology as described in Beimforde et al. (2014), Hongsanan et al. (2016) and Pérez-Ortega et al. (2016) for dating molecular clock analyses. Estimating divergences time of the common ancestor of Palawaniaceae in Dothideomycetes was performed using BEAST analysis.

A molecular clock tree was generated using the calibration points from Beimforde et al. (2014), Hongsanan et al. (2016) and Pérez-Ortega et al. (2016). Metacapnodiaceae was used as a minimum age of Capnodiales (normal distribution, mean $=100, \mathrm{SD}=150, \mathrm{CI}=400$ ) with Calicium (Rikkinen 2003) (gamma distribution shape $=1.0$, scale $=70$, offset $=35$ ) and Microthyrium (gamma distribution scale $=70$, offset $=35$ ) for calibrations in this study.

\section{Molecular clock analysis}

Molecular clock analyses were performed using BEAST 1.8.0 (Drummond et al. 2012) following the methodology as described in Hongsanan et al. (2016). LSU, SSU and RPB2 aligned sequences data partitions were prepared separately. Each data partitions were loaded to BEAUti 1.8.0 (Drummond et al. 2012). GTR $+\mathrm{I}+\mathrm{G}$ model were selected for $\mathrm{LSU}$, and $\mathrm{TrN}+\mathrm{I}+\mathrm{G}$ model for SSU and RPB2 based on the results from jModelTest 2.1.10 (Darriba et al. 2012). Taxa sets were prepared for the most recent common ancestor (TMRCA) and other interesting groups. A lognormal relaxed clock (uncorrelated) model was selected, and a birth/death incomplete sampling tree prior was used to model the speciation of nodes in the topology.

BEAST analyses were run for 50 million generations, and sampling parameters every 1000 generations. Convergence, mixing and effective sample sizes $(\mathrm{ESS}>200)$ of parameters were checked using Tracer v1.6 (Rambaut et al. 2014). The first 50,000 trees representing the burn-in phase were discarded. The remaining trees were combined using LogCombiner 1.8.0 and generate a maximum clade credibility tree (MCC) with TreeAnnotator 1.8.0. Molecular clock trees were drawn using FigTree v. 1.4.0 (Rambaut 2009).

The ages for geological time periods have taken from the most recent International Chronostratigraphic Chart (v2016/04) by International Commission on Stratigraphy (IUGS) and available from www.stratgraphy.org, are indicated at the base of the evolution tree (Fig. 2)

\section{Results \\ Phylogenetic analysis}

The combined of LSU, SSU and RPB2 sequence data (93 taxa) including our new strains were analyzed by maximum likelihood (ML) and Bayesian analyses. Representative stains from Lecanoromycetes were selected as outgroup taxa. A best scoring RAxML analysis is shown in Fig. 1. The trees generated from both methods are similar in topology with no significant difference. In the phylogenetic tree (Fig. 1) our new strains clustered within Dothideomycetes, and as a sister clade with Pleurotremataceae with moderate support $(62 \% \mathrm{ML})$ and related to the order Muyocopronales with 93\% ML, 1.0 PP support.

\section{Molecular clock and Divergence time estimates}

The maximum clade credibility (MCC) tree indicates the divergence estimates at 319 Mya (247-400), in the Carboniferous for Dothideomycetes and Lecanomycetes. Divergence time estimates in this study for the target groups and the splits between them are shown in Table 2. A simplified of diagrammatic scheme representation for the target groups is show in Fig. 3.

Acrospermales has an estimated crown date in the Cretaceous of 124 Mya (90-160). The family shares the most common ancestor with Pleurotremataceae, Palawaniaceae and Muyocopronales at 215 Mya (164-272) in the late Triassic. Pleurotremataceae has an estimated crown date in the early Cenozoic of 55 Mya (38-74) and shares the most common ancestor with Palawaniaceae and Muyocopronales in the early Jurassic at 192 Mya (145-243). The split between Muyocopronales which was introduced as a new order in Mapook et al. (2016b) and Palawaniaceae (in this study) is estimated at 172 Mya (130-218), during the Jurassic with estimated crown dates of Muyocopronales during the Cenozoic at 52 Mya (38-66). 
Table 1 Taxa used in this study and their GenBank accession numbers. New sequences are in bold.

\begin{tabular}{|c|c|c|c|c|}
\hline \multirow{2}{*}{ Taxon } & \multirow{2}{*}{ Culture accession no. } & \multicolumn{3}{|c|}{ GenBank accession no. } \\
\hline & & LSU & SSU & RPB2 \\
\hline Acrospermum adeanum & M133 & EU940104 & EU940031 & EU940320 \\
\hline Acrospermum compressum & M151 & EU940084 & EU940012 & EU940301 \\
\hline Acrospermum gramineum & M152 & EU940085 & EU940013 & EU940302 \\
\hline Alternaria alternata & KFRD-18 & KX609781 & KX609769 & - \\
\hline Alternariaster bidentis & CBS 134021 & КС609341 & - & KC609347 \\
\hline Antennariella placitae & CBS: 124785 & GQ303299 & - & - \\
\hline Asterina fuchsiae & TH590 & GU586216 & GU586210 & - \\
\hline Asterina phenacis & TH589 & GU586217 & GU586211 & - \\
\hline Bambusicola massarinia & MFLUCC 11-0389 & JX442037 & JX442041 & KU940169 \\
\hline Bambusicola splendida & MFLUCC 11439 & JX442038 & JX442042 & - \\
\hline Botryosphaeria agaves & MFLUCC 11-0125 & JX646808 & JX646825 & - \\
\hline Botryosphaeria tsugae & AFTOL-ID 1586 & DQ767655 & - & DQ767644 \\
\hline Calicium viride & 10-VII-1997 (DUKE) & AF356670 & AF356669 & AY641031 \\
\hline Calicium salicinum & CBS 100898 & KF157982 & KF157970 & KF157998 \\
\hline Camarosporium quaternatum & CBS 483.95 & GU301806 & GU296141 & GU357761 \\
\hline Capnodium salicinum & AFTOL-ID 937 & DQ678050 & DQ677997 & - \\
\hline Caryospora minima & - & EU196550 & EU196551 & - \\
\hline Chaetothyriothecium elegans & CPC 21375 & KF268420 & - & - \\
\hline Corynespora cassiicola & CBS 100822 & GU301808 & GU296144 & GU371742 \\
\hline Corynespora smithii & CABI 5649b & GU323201 & - & GU371783 \\
\hline Cucurbitaria berberidis & MFLUCC 11-0387 & KC506796 & KC506800 & - \\
\hline Cyphelium tigillare & Tibell 22343 (UPS) & AY453641 & AF241545 & - \\
\hline Cyphelium inquinans & Tibell 22283 (UPS) & AY453639 & U86695 & - \\
\hline Cystocoleus ebeneus & L161 & EU048578 & EU048571 & - \\
\hline Didymella exigua & CBS 183.55 & JX681089 & EU754056 & GU371764 \\
\hline Didymosphaeria rubi-ulmifolii & MFLUCC 14-0023 & KJ436586 & KJ436588 & - \\
\hline Dothiora cannabinae & AFTOL ID 1359 & DQ470984 & DQ479933 & DQ470936 \\
\hline Elsinoe fawcettii & CPC 18535 & JN940382 & JN940559 & - \\
\hline Elsinoe verbenae & CPC 18561 & JN940391 & JN940562 & - \\
\hline Extremus antarcticus & CCFEE 5312 & KF310020 & - & KF310086 \\
\hline Helicascus nypae & BCC 36751 & GU479788 & GU479754 & GU479826 \\
\hline Julella avicenniae & BCC 20173 & GU371822 & GU371830 & GU371786 \\
\hline Karschia cezannei & Cezanne-Eichler B26 & KP456152 & - & - \\
\hline Katumotoa bambusicola & KT $1517 \mathrm{a}$ & AB524595 & AB524454 & AB539095 \\
\hline Labrocarpon canariense & Ertz 16907 (BR) & KP456157 & - & - \\
\hline Lentithecium fluviatile & CBS 123090 & FJ795450 & FJ795492 & FJ795467 \\
\hline Leptosphaeria doliolum & MFLUCC 15-1875 & KT454719 & KT454734 & - \\
\hline Leptosphaerulina australis & CBS 317.83 & EU754166 & GU296160 & GU371790 \\
\hline Leptoxyphium cacuminum & MFLUCC10-0049 & JN832602 & JN832587 & - \\
\hline Lophiotrema nucula & CBS 62786 & GU301837 & GU296167 & GU371792 \\
\hline Lophium mytilinum & AFTOL-ID 1609 & DQ678081 & DQ678030 & DQ677979 \\
\hline Massarina bambusina & H 4321 & AB807536 & AB797246 & - \\
\hline Massarina eburnea & CBS 473.64 & GU301840 & GU296170 & GU371732 \\
\hline Melanomma pulvis-pyrius & CBS 37175 & GU301845 & FJ201989 & GU371798 \\
\hline Melaspileopsis cf. diplasiospora & Ertz 16247 (BR) & KP456164 & - & - \\
\hline
\end{tabular}




\begin{tabular}{|c|c|c|c|c|}
\hline \multirow{2}{*}{ Taxon } & \multirow{2}{*}{ Culture accession no. } & \multicolumn{3}{|c|}{ GenBank accession no. } \\
\hline & & LSU & SSU & RPB2 \\
\hline Microsphaeropsis olivacea & CBS 23377 & GU237988 & - & KT389643 \\
\hline Microthyrium microscopicum & CBS 115976 & GU301846 & GU296175 & GU371734 \\
\hline Microthyrium buxicola & MFLUCC 15-0213 & KT306552 & KT306550 & - \\
\hline Murispora rubicunda & IFRD 2017 & FJ795507 & GU456308 & - \\
\hline Muyocopron castanopsis & MFLUCC 10-0042 & - & JQ036225 & - \\
\hline Muyocopron castanopsis & MFLUCC 14-1108 & KU726965 & KU726968 & KY225778 \\
\hline Muyocopron dipterocarpi & MFLUCC 14-1103 & KU726966 & KU726969 & KY225779 \\
\hline Muyocopron lithocarpi & MFLUCC 10-0041 & JQ036230 & JQ036226 & - \\
\hline Muyocopron lithocarpi & MFLUCC 14-1106 & KU726967 & KU726970 & KY225780 \\
\hline Myriangium duriaei & CBS 260.36 & NG_027579 & AF242266 & KT216528 \\
\hline Myriangium hispanicum & CBS 247.33 & GU301854 & GU296180 & GU371744 \\
\hline Mytilinidion rhenanum & CBS 135.34 & FJ161175 & FJ161136 & FJ161115 \\
\hline Natipusilla decorospora & AF236 1a & HM196369 & HM196376 & - \\
\hline Natipusilla naponensis & $\mathrm{AF} 2171 \mathrm{a}$ & HM196371 & HM196378 & - \\
\hline Neocylindroseptoria pistaciae & CBS 471.69 & KF251656 & - & KF252161 \\
\hline Multiseptospora thailandica & MFLUCC 11-0183 & KP744490 & KP753955 & - \\
\hline Phaeodimeriella cissampeli & MFLU 16-0558 & KU746806 & KU746808 & KU746810 \\
\hline Phaeodimeriella dilleniae & MFLU 14-0013 & KU746805 & KU746807 & KU746809 \\
\hline Phaeotrichum benjaminii & CBS 541.72 & AY004340 & AY016348 & GU357788 \\
\hline Physcia aipolia & AFTOL-ID 84 & DQ782904.1 & DQ782876 & DQ782862 \\
\hline Piedraia hortae & CBS 480.64 & GU214466 & - & - \\
\hline Palawania thailandense & MFLUCC 14-1121 & KY086493 & KY086495 & KY086496 \\
\hline Palawania thailandense & MFLU 16-1871 & KY086494 & - & - \\
\hline Platystomum crataegi & MFLUCC 14-0925 & KT026109 & KT026113 & - \\
\hline Pleomassaria siparia & AFTOL-ID 1600 & DQ678078 & DQ678027 & DQ677976 \\
\hline Pleospora herbarum & IT 956 & KP334709 & KP334729 & KP334733 \\
\hline Pleurotrema rhizophorae & JK 5456A & GU479799 & - & - \\
\hline Pleurotrema rhizophorae & BCC15481 & - & KF160009 & \\
\hline Pleurotrema tiomanensis & NTOU3636 & KC692156 & KC692155 & - \\
\hline Preussia funiculata & CBS 659.74 & GU301864 & GU296187 & GU371799 \\
\hline Pseudomassariosphaeria bromicola & IT-1333 & KT305994 & KT305996 & - \\
\hline Pseudostrickeria muriformis & MFLUCC 13-0764 & KT934254 & KT934258 & - \\
\hline Ramularia endophylla & CBS 113265 & KF251833 & - & KP894673 \\
\hline Rasutoria pseudotsugae & rapssd & EF114704 & EF114729 & - \\
\hline Rasutoria tsugae & ratstk & EF114705 & EF114730 & - \\
\hline Salsuginea ramicola & KT 2597.1 & GU479800 & GU479768 & GU479833 \\
\hline Schizothyrium pomi & CBS 406.61 & EF134949 & - & KF902384 \\
\hline Stictographa lentiginosa & Ertz $17570(\mathrm{BR})$ & KP456170 & - & - \\
\hline Sympoventuria capensis & CBS 120136 & KF156104 & KF156094 & - \\
\hline Teratosphaeria fibrillosa & CBS 121707 & GU323213 & GU296199 & GU357767 \\
\hline Trichodelitschia munkii & Kruys 201 (UPS) & DQ384096 & DQ384070 & - \\
\hline Uwebraunia commune & $\mathrm{NC} 132 \mathrm{C} 1 \mathrm{~d}$ & - & - & KT216546 \\
\hline Venturia inaequalis & CBS 594.70 & GU301879 & GU296205 & GU357757 \\
\hline Xenolophium applanatum & CBS 123127 & GU456330 & GU456313 & GU456355 \\
\hline Zeloasperisporium wrightiae & MFLUCC 15-0225 & KT387737 & KT387738 & - \\
\hline Zeloasperisporium hyphopodioides & CBS 218.95 & EU035442 & - & - \\
\hline Zeloasperisporium siamense & IFRDCC 2194 & JQ036228 & JQ036223 & - \\
\hline
\end{tabular}


Table 2 Divergence time estimates of the target groups in this study

\begin{tabular}{lll}
\hline & Geological period & Time (Mya) \\
\hline Acrospermales crown group & Cretaceous & $124(90-160)$ \\
\hline Muyocopronales crown group & $\begin{array}{l}\text { Cenozoic Era, Tertiary, } \\
\text { Paleogene }\end{array}$ & $52(38-66)$ \\
\hline Pleurotremataceae crown group & $\begin{array}{l}\text { Cenozoic Era, Tertiary, } \\
\text { Paleogene }\end{array}$ & $55(38-74)$ \\
\hline Palawaniaceae + Muyocopronales & Jurassic & $172(130-218)$ \\
\hline
\end{tabular}

\section{Taxonomy}

Palawaniaceae Mapook \& K.D. Hyde, fam. nov. Index Fungorum number: IF552528, Facesoffungi number: FoF 02653

Type genus: Palawania Syd. \& P. Syd., Philipp. J. Sci., C, Bot. 9(2): 171 (1914).

Saprobic on surface of dead rachides and spines of Arecaceae, rarely on leaves, at first appearing as solitary, circular, black spots, then being confluent, superficial hyphae absent. Sexual morph: Ascomata superficial, scattered, or some solitary, flattened, sub-carbonaceous, dark brown to black, basal layer poorly developed, with a central ostiole. Peridium wide, upper wall comprising of dark brown or black to light brown cell of textura epidermoidea, with light brown cells of textura angularis at the sides. Hamathecium comprising asci with cylindrical to filiform, septate, pseudoparaphyses which are longer than the asci and inclined toward the central ostiole. Asci 8-spored, bitunicate, inequilateral to ovoid, pedicellate, straight or slightly curved, short pedicellate, without ocular chamber. Ascospores overlapping, irregularly arranged, oblong to broadly fusiform, hyaline, becoming brown at maturity, 1-septate, constricted at the septum, guttulate, surrounded by hyaline, gelatinous sheath, observed clearly when mounted in Indian ink. Asexual morph: Undetermined.

Notes - Based on phylogenetic analysis, Palawaniaceae forms a distinct family in the clade comprising Acrospermales, Pleurotremataceae and Muyocopronales (Fig. 1). Palawaniaceae differs from Acrospermales and Pleurotremataceae (= Dyfrolomycetaceae) in morphology (Riddle 1920, Minter et al. 2007, Hyde et al. 2013, Pang et al. 2013, Maharachchikumbura et al. 2016, Mapook et al. 2016b). Although, the new family has similarity with Muyocopronales in its superficial, flattened ascomata, and pseudoparaphyses that are longer than the asci, they differ in peridium wall patterns.

Palawania Syd. \& P. Syd., Philipp. J. Sci., C, Bot. 9(2): 171 (1914).

Saprobic on dead palm rachides. Sexual morph: Ascomata superficial, solitary or scattered, coriaceous, appearing as circular, scattered, flattened, dark brown to black spots, covering the host, without a subiculum, with a poorly developed basal layer and an irregular margin. Ostiole central. Peridium 10-50 $\mu \mathrm{m}$ wide, comprising of dark brown or black to light brown cell of textura epidermoidea at the top, with light brown cells of textura angularis at the sides. Hamathecium comprising 1.5-2.5 $\mu \mathrm{m}$ wide, cylindrical to filiform, septate, pseudoparaphyses. Asci 8-spored, bitunicate, inequilateral to ovoid, pedicellate, straight or slightly curved, short pedicel, without ocular chamber. Ascospores overlapping, irregularly arranged, oblong to broadly fusiform, hyaline, becoming brown at maturity, 1-septate, constricted at the septum, guttulate, surrounded by hyaline gelatinous sheath, observed clearly when mounted in Indian ink. Asexual morph: Undetermined.

Type species - Palawania grandis (Niessl) Syd. \& P. Syd., Philipp. J. Sci., C, Bot. 9(2):

Notes - The type specimen of Palawania grandis was first reported from Calamus sp. 


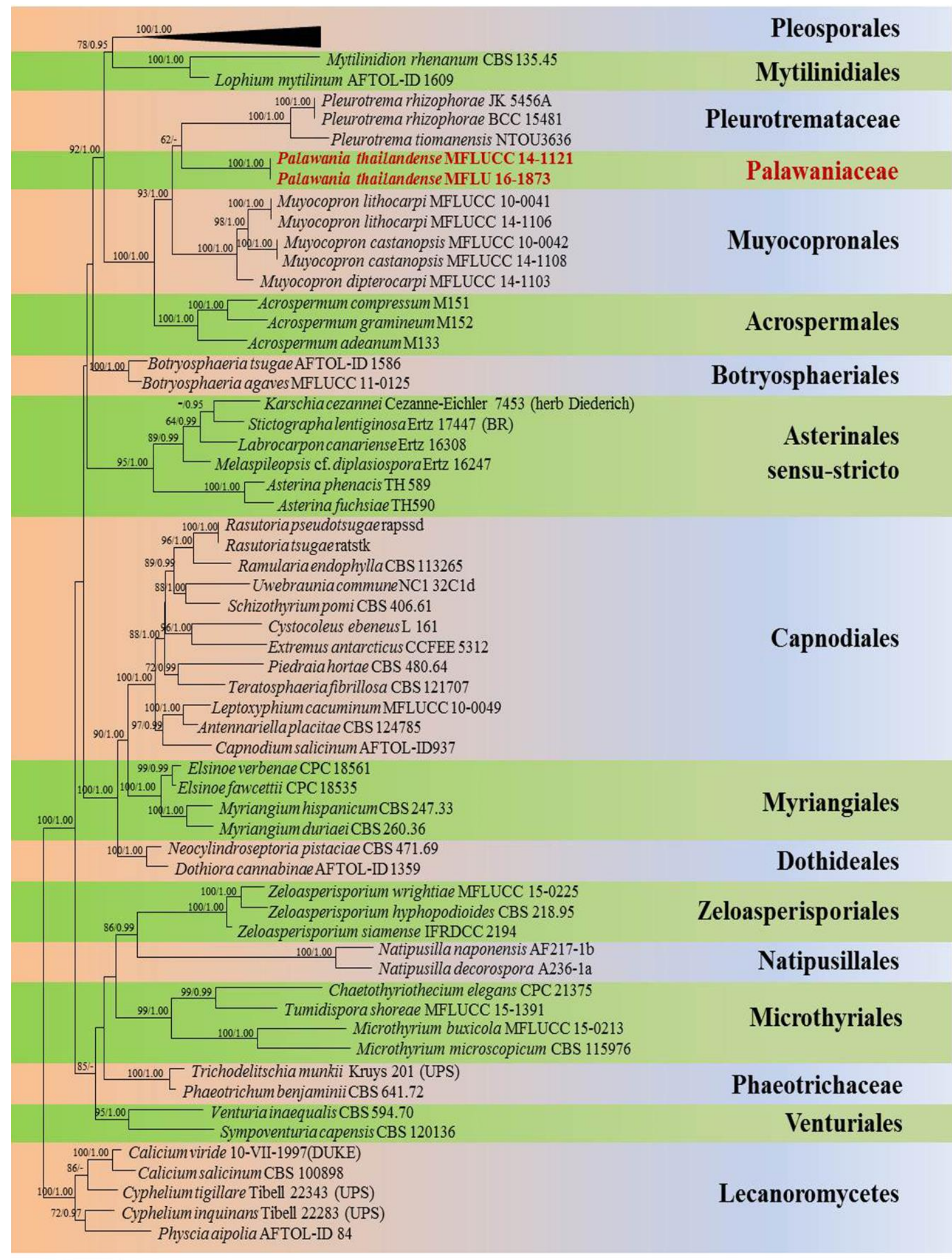

0.2

Fig 1 - Phylogram generated from RAxML based on combined LSU, SSU and RPB2 sequence data to establish the new family Palawaniaceae. Bootstrap support values for maximum likelihood (ML) greater than $60 \%$ and Bayesian posterior probabilities (PP) equal to or greater than 0.95 are given above the nodes. The new isolates are in red. The tree is rooted with Lecanomycetes. 


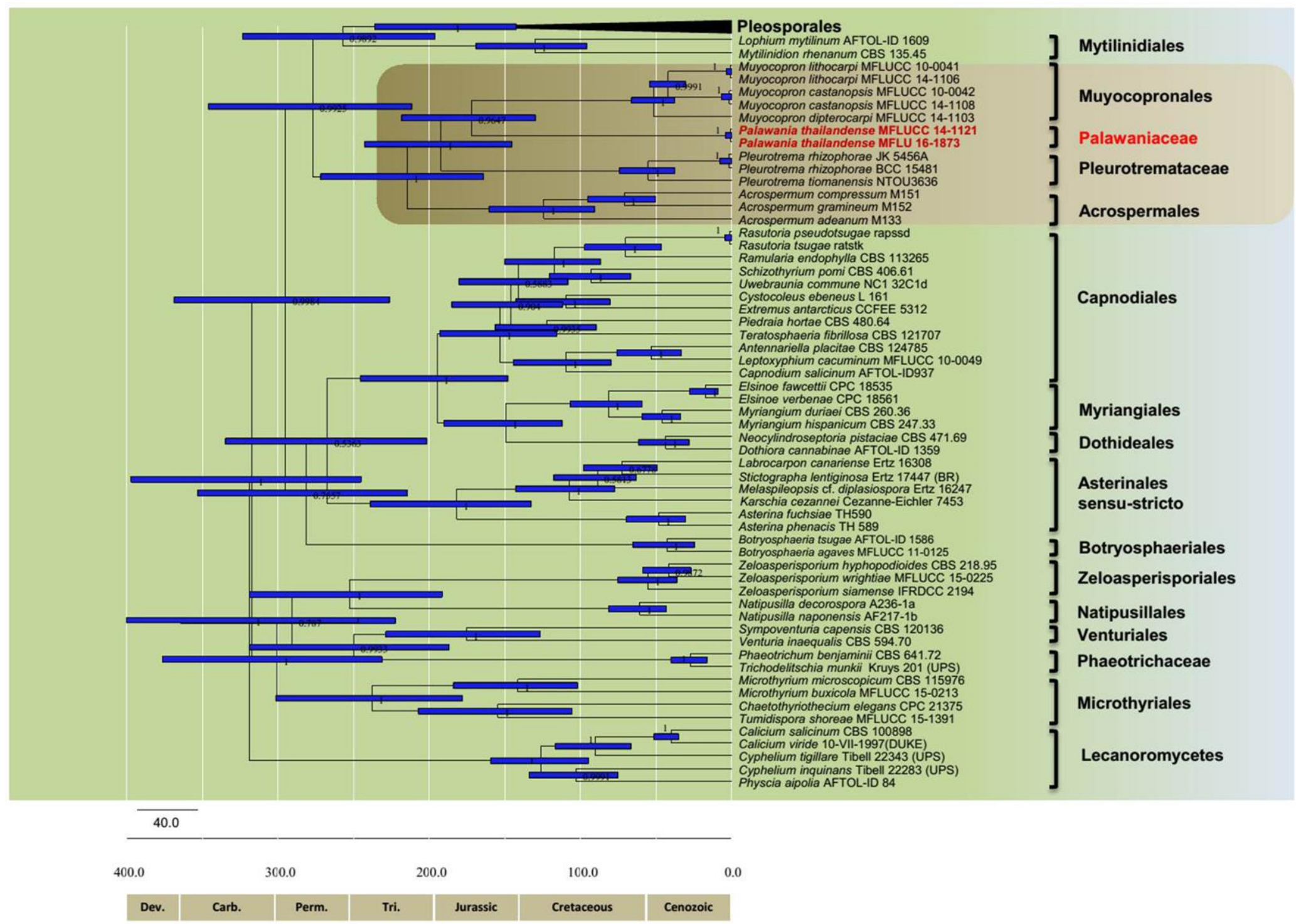

Fig 2 - Divergence time estimates from Dothideomycetes obtained from a Bayesian approach (BEAST). Bars correspond to the 95\% highest posterior density (HPD) intervals. The fossil minimum age constraints and second calibrations used in this study are marked with red dots. Geological periods are indicated at the base of the tree and abbreviated as: Dev. $=$ Devonian, Carb. $=$ Carboniferous, Perm. $=$ Permian, Tri. $=$ Triassic. The Palawania species discussed in this study are highlighted in red. 


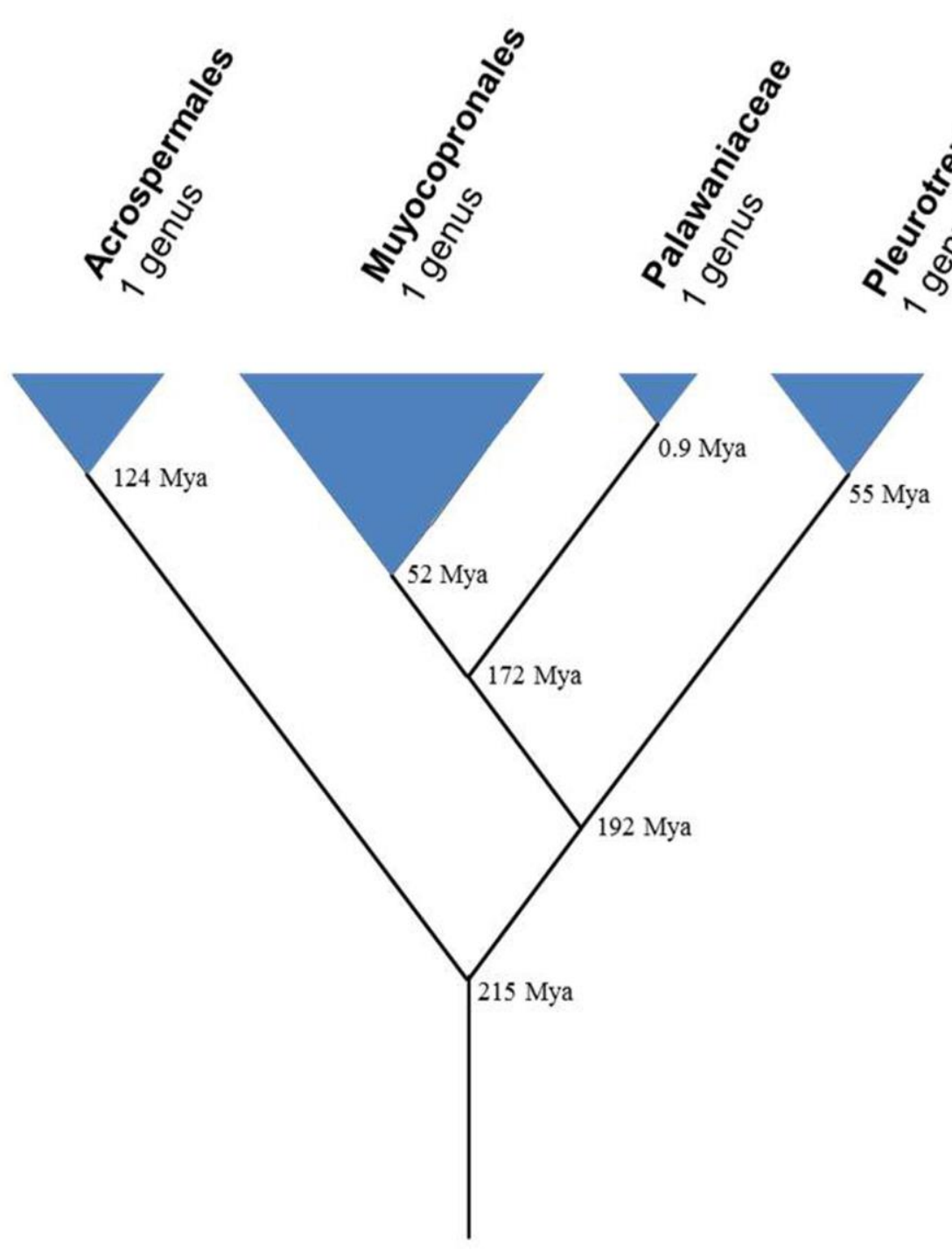

Fig 3 - A simplified of diagrammatic scheme of the evolution representation for the target groups. Divergence time estimates are given at the branches as millions of years ago (Mya).

Palawania thailandense Mapook \& K.D. Hyde, sp. nov.

Index Fungorum number: IF552527, Facesoffungi number: FoF 02654

Etymology - Name reflects the country from where this species was collected, Thailand

Holotype - MFLU 16-1872

Saprobic on dead rachis of Dypsis lutescens (H. Wendl.) Beentje \& J. Dransf. Sexual morph: Ascomata 75-115(-125) $\mu \mathrm{m}$ high $\times(400-) 475-495(-575) \mu \mathrm{m}$ diam. $(\bar{x}=105 \times 485 \mu \mathrm{m}$, $\mathrm{n}=5$ ), superficial, solitary or scattered, coriaceous, appearing as circular, scattered, flattened, dark brown to black spots, covering the host, without a subiculum, with a poorly developed basal layer and an irregular margin. Ostiole central. Peridium (15-)20-40 $\mu \mathrm{m}$ wide, comprising dark brown or black to light brown cells of textura epidermoidea from above, with light brown cells of textura angularis at the sides. Hamathecium comprising 1.5-2.5 $\mu \mathrm{m}$ wide, cylindrical to filiform, septate, pseudoparaphyses. Asci (60-)80-110 × 20-30 $\mu \mathrm{m}(\bar{x}=85 \times 25 \mu \mathrm{m}, \mathrm{n}=20), 8$-spored, bitunicate, inequilateral to ovoid, pedicellate, straight or slightly curved, without ocular chamber. Ascospores 20-27 $\times(6-) 7-10 \mu \mathrm{m}(\bar{x}=25 \times 8 \mu \mathrm{m}, \mathrm{n}=40)$, overlapping, irregularly arranged, oblong to 
broadly fusiform, hyaline, 1-septate, constricted at the septum, guttulate, surrounded by hyaline gelatinous sheath, observed clearly when mounted in Indian ink. Asexual morph: Undetermined.

Material examined - THAILAND, Chiang Rai, Chiang Rai Horticultural Research Center, on dried twigs of Dypsis lutescens (Arecaceae), 9 September 2014, A. Mapook, (MFLU 16-1872, holotype), ex-type living culture MFLUCC 14-1121, (isotype in HKAS, under the code of HKAS 95075), on dried twigs of Phoenix roebelenii (Arecaceae), 9 September 2014, A. Mapook, (MFLU 16-1873, HKAS 95076, paratype).

Notes - Palawania thailandense (MFLU 16-1872) is a saprobe on dead rachis of Dypsis lutescens. Our collection is most similar to Palawania grandis in size and shape of ascomata and ascospores (Table 3), but it differs in having light brown to brown thyriothecia, the peridium radiating cell pattern and longer asci. Thus, we consider our taxon is a new species based on morphology.

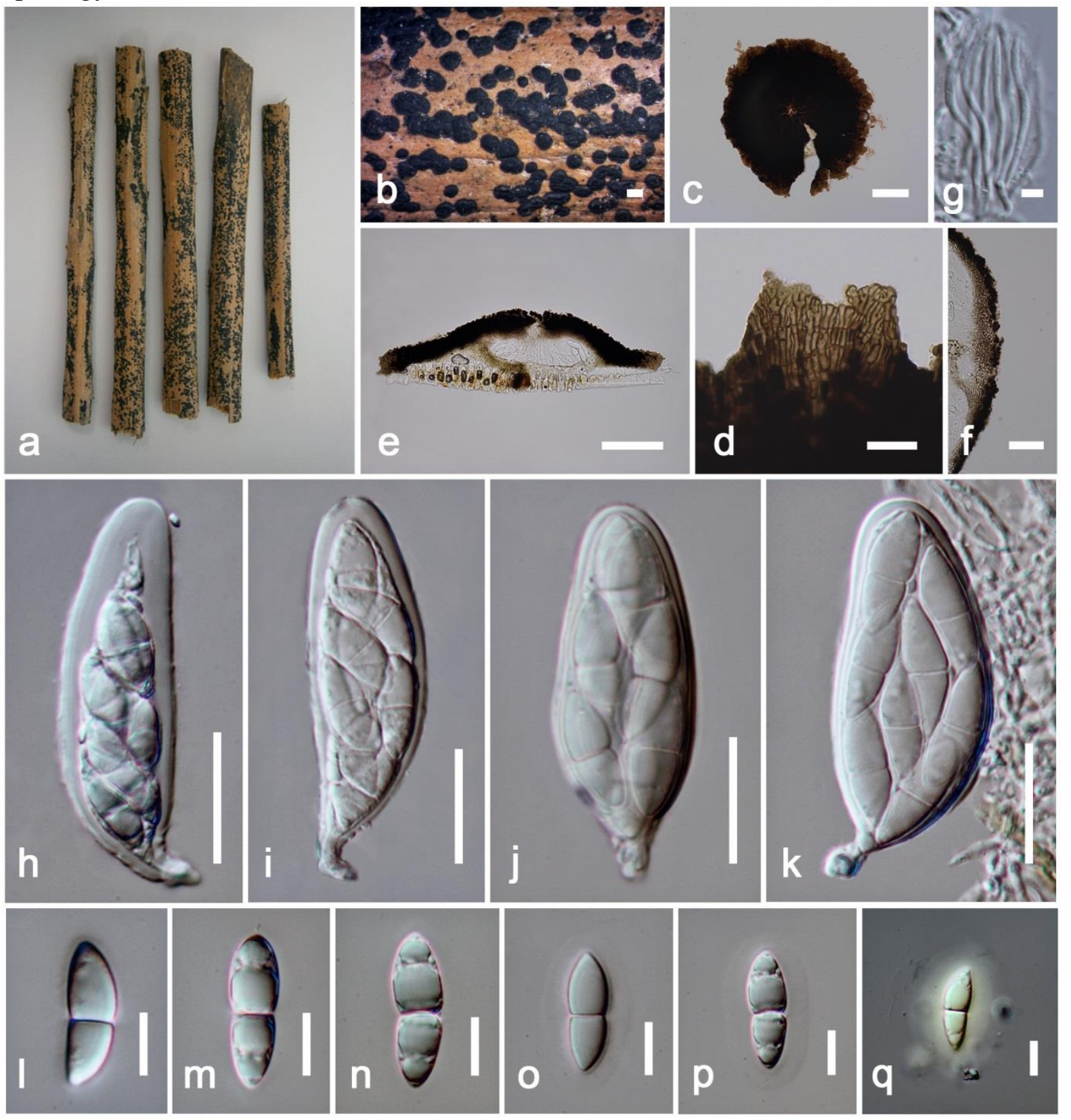

Fig 4 - Palawania thailandense (holotype). a, b Superficial ascomata on substrate. c, d Squash mounts showing ascomata walls. e Section of ascoma. f Peridium. g Pseudoparaphyses. $\mathbf{h}-\mathbf{k}$ Asci. l-p Ascospores. q Ascospores surrounded by hyaline gelatinous sheath in Indian ink. - Bars: $b=$ $500 \mu \mathrm{m}, \mathrm{c}, \mathrm{e}=100 \mu \mathrm{m}, \mathrm{f}, \mathrm{h}-\mathrm{k}=20 \mu \mathrm{m}, \mathrm{d}, \mathrm{l}-\mathrm{q}=10 \mu \mathrm{m}, \mathrm{g}=5 \mu \mathrm{m}$ 
Table 3 Synopsis of Palawania species with some similar morphological features.

\begin{tabular}{|c|c|c|c|c|c|c|}
\hline Species & Ascomata & $\begin{array}{l}\text { Asci } \\
\text { size } \\
(\mu \mathrm{m})\end{array}$ & $\begin{array}{l}\text { Ascospores } \\
\text { size } \\
(\mu \mathrm{m}) \\
\end{array}$ & Septation & $\begin{array}{l}\text { Host } \\
\text { association } \\
\text { family }\end{array}$ & $\begin{array}{l}\text { Source } \\
\text { references }\end{array}$ \\
\hline $\begin{array}{l}\text { Palawania grandis } \\
\text { (Niessl) Syd. \& P. Syd. } \\
1914\end{array}$ & $\begin{array}{l}86-110 \mu \mathrm{m} \\
\text { high } \times 310- \\
480 \mu \mathrm{m} \\
\text { diam. }\end{array}$ & $\begin{array}{l}80-86 \times \\
24-30\end{array}$ & $\begin{array}{l}26-31 \times 9- \\
15, \text { fusiform } \\
\text { to ellipsoid- } \\
\text { fusiform, } \\
\text { brown when } \\
\text { mature }\end{array}$ & $\begin{array}{l}\text { 1-septate, } \\
\text { surrounde } \\
\text { d by a } \\
\text { hyaline } \\
\text { sheath. }\end{array}$ & $\begin{array}{l}\text { Calamus sp. } \\
\text { (Arecaceae) }\end{array}$ & $\begin{array}{l}\text { Wu et al. } 2011 . \\
\text { (M-0155393, } \\
\text { F8214, } \\
\text { F90149, } \\
\text { F8873) }\end{array}$ \\
\hline $\begin{array}{l}\text { Palawania grandis } \\
\text { (Niessl) Syd. \& P. Syd. } \\
1914\end{array}$ & $\begin{array}{l}65-93 \mu \mathrm{m} \\
\text { high } \times 330- \\
560 \mu \mathrm{m} \\
\text { diam. }\end{array}$ & $\begin{array}{l}65-85 \times \\
20-22.5\end{array}$ & $\begin{array}{l}22.5-27 \times \\
8-10\end{array}$ & 1-septate & $\begin{array}{l}\text { Calamus sp. } \\
\text { (Arecaceae) }\end{array}$ & $\begin{array}{l}\text { Batista \& Vital } \\
1960\end{array}$ \\
\hline $\begin{array}{l}\text { Palawania cocois Syd. } \\
\text { \& P. Syd. } 1914\end{array}$ & $\begin{array}{l}0.3-1(8) \\
\mathrm{mm}\end{array}$ & $\begin{array}{l}50-75 \times \\
20-26\end{array}$ & $\begin{array}{l}22-24 \times 8- \\
10\end{array}$ & 1-septate & $\begin{array}{l}\text { Cocos } \\
\text { nucifera } \mathrm{L} . \\
\text { (Arecaceae) }\end{array}$ & $\begin{array}{l}\text { Syd. \& P. Syd. } \\
1914\end{array}$ \\
\hline $\begin{array}{l}\text { Palawania } \\
\text { thailandense } \\
\text { (MFLUCC 14-1121) }\end{array}$ & $\begin{array}{l}75-115(- \\
125) \mu \mathrm{m} \\
\text { high } \times \\
(430-) 475- \\
495 \mu \mathrm{m} \\
\text { diam. }\end{array}$ & $\begin{array}{l}(60- \\
) 80-110 \\
\times 22-25\end{array}$ & $\begin{array}{l}22-27 \times 7- \\
10\end{array}$ & $\begin{array}{l}\text { 1-septate, } \\
\text { surrounde } \\
\text { d by a } \\
\text { hyaline } \\
\text { sheath. }\end{array}$ & $\begin{array}{l}\text { Dypsis } \\
\text { lutescens } \\
\text { (Arecaceae) }\end{array}$ & This study \\
\hline
\end{tabular}

\section{Discussion}

The genus Palawania has been placed in the family Microthyriaceae (Müller \& von Arx 1962, Lumbsch \& Huhndorf 2010, Wu et al. 2011, Hyde et al. 2013, Wijayawardene et al. 2014), based on morphology. Wu et al. (2011) re-examined the type material (P. grandis (Niessl) Syd. \& P. Syd) and provided descriptions and illustrations. However, fresh collections and sequence data are required to confirm the phylogenetic placement of Palawania (Wu et al. 2011). In this study, two fresh collections were made from the same location associated with palms (Arecaceae). The collections are similar to Palawania and are morphologically and phylogenetically the same genus (Fig. 1). However, morphological comparisons within other Palawania species show that our collections differ and we introduce our collection as a new species. Palawania cocois and $P$. grandis were listed under Microthyriales (Index Fungorum 2016), both being common on Arecaceae. Palawania grandis is morphologically most similar with our new species in the size and shape of its ascomata and ascospores (Wu et al. 2011). However, it differs in having light brown to brown thyriothecia, in the peridium radiating cell pattern and longer asci.

Based on phylogenetic analyses, we conclude that the genus Palawania cannot be placed in Microthyriales, as the genus formed a sister clade with Pleurotremataceae and has a close phylogenetic relationship with Muyocopronales and Acrospermales (Fig. 1). Species of Acrospermales and Pleurotremataceae are morphologically very different from Palawania species. Therefore, we introduce a new family Palawaniaceae to accommodate this group of unique fungi. The new family is ecologically similar to Muyocopronales and shares some morphological characters, but differs in its peridium wall pattern, shape of asci and ascospores that are surrounded by hyaline gelatinous sheath.

Several of divergence times estimates in Fungi have been published with available fossil evidence from ascomycetes (Vijaykrishna et al. 2006, Gueidan et al. 2008, Schoch et al. 2009, Gazis et al. 2012, Prieto et al. 2013, Beimforde et al. 2014, Hongsanan et al. 2016 and PérezOrtega et al. 2016), and events in the geological record and their correlations with fungal fossils have been reported in Berbee and Taylor (2010). The fossil fungal spore record was associated with the Permian-Triassic extinction event (252 Mya) (Visscher et al. 1996, Shen et al. 2011). During this period, the numbers of marine and terrestrial fungi are through to have increased (Visscher et al. 1996). More than $95 \%$ of land plants however, disappeared (Labandiera and 
Sepkoski 1993, Retallack 1995, Eshet et al. 1995). Furthermore, 34\% of marine genera disappeared, land vertebrates and many of amphibians also became extinct after the TriassicJurassic extinction (201.3 Mya) according to Benton (1988) and Ryder et al. (1996).

Sequence data from Palawania thailandense cluster in the new family Palawaniaceae. The maximum clade credibility (MCC) tree with divergence estimates using three calibration points (Fig. 2) is topologically quite similar to the Bayesian and ML analyses in most of the major lineages (Fig. 1). A difference occurred in the clade comprising our target group, with the families, Acrospermaceae (Acrospermales), Muyocopronaceae (Muyocopronales), Palawaniaceae (Dothideomycetes family, incertae sedis), and Pleurotremataceae (Dothideomycetes family, incertae sedis), which are generally poorly-studied and lack molecular data. In the maximum likelihood analysis (Fig. 1), Palawaniaceae shared the most common ancestor with Pleurotremataceae, while the MCC tree provided by BEAST suggests that Palawaniaceae is closely related and shared the most common ancestor with Muyocopronales having diverged in the Jurassic period (approximately 172 Mya), which was around 29 Mya after the Triassic-Jurassic extinction (201.3 Mya). However, the relationship between Palawaniaceae and Pleurotremataceae in the ML tree is moderately supported. The relationship between Palawaniaceae and Muyocopronaceae is well-supported in the MCC tree, and these families share many characters. Thus, we believe that the new family is most closely related to Muyocopronaceae and should probably be placed in the order Muyocopronales.

Acknowledgements Ausana Mapook is grateful to Research and Researchers for Industries (RRI) PHD57I0012 under the Thailand Research Fund for providing financial support. K.D. Hyde is visiting Professor at King Saud University and thanks the Chinese Academy of Sciences, project number 2013T2S0030, for the award of Visiting Professorship for Senior International Scientists at Kunming Institute of Botany. Saranyaphat Boonmee thanks the National Research Council of Thailand, project number 2560A30702021 and the Thailand Research Fund, project number TRG5880152 for providing financial support. Chayanard Phukhamsakda thanks the Royal Golden Jubilee PhD Program under the Thailand Research Fund for the award of a scholarship number PHD/0020/2557 for study towards a PhD. We thank the Plant Germplasm and Genomics Center in Germplasm Bank of Wild Species, Kunming Institute of Botany for the help with the molecular work. Ausana Mapook also thanks to Dr. Shaun Pennycook, Rungtiwa Phookamsak, Mingkwan Doilom, Saowaluck Tibpromma, Dhanushka N. Wanasinghe, Chada Norphanphoun, Sirinapa Konta, J.K. Lui for their valuable suggestions and help.

\section{References}

Batista AC, Vital AF. 1960 - A revision of some species of the genera Melanoplaca Syd, Mendogia Racib. and Palawania Syd. Atas do Instituto de Micologia. Universidade Federal de Pernambuco, Recife 112, 79-92.

Beimforde C, Feldberg K, Nylinder S, Rikkinen J et al. 2014 - Estimating the Phanerozoic history of the Ascomycota lineages: combining fossil and molecular data. Molecular Phylogenetics and Evolution 78, 386-398.

Benton MJ. 1988 - Mass extinctions and the fossil record of reptiles: paraphyly, patchiness and periodicity. Systematics Association special 34, 269-294.

Berbee ML, Taylor JW. 2010 - Dating the molecular clock in fungi - how close are we? Fungal Biology Reviews 24, 1-16.

Chomnunti P, Hongsanan S, Hudson BA, Tian Q et al. 2014 - The sooty moulds. Fungal Diversity $66,1-36$.

Darriba D, Taboada GL, Doallo R, Posada D. 2012 - jModelTest 2: more models, new heuristics and parallel computing. Nature Methods 9, 772-772.

Drummond AJ, Suchard MA, Xie D, Rambaut A. 2012 - Bayesian Phylogenetics with BEAUti and the BEAST 1.7. Molecular Biology and Evolution 29, 1969-1973. 
Eshet Y, Rampino MR, Visscher H. 1995 - Fungal event and palynological record of ecological crisis and recovery across the Permian-Triassic boundary. Geology 23, 967-970.

Gazis R, Miadlikowska J, Lutzoni F, Arnold AE, Chaverri P. 2012 - Culture-based study of endophytes associated with rubber trees in Peru reveals a new class of Pezizomycotina: Xylonomycetes. Molecular Phylogenetics and Evolution 65, 294-30.

Gueidan C, Ruibal CV, de Hoog GS, Gorbushina AA et al. 2008 - An extremotolerant rockinhabiting ancestor for mutualistic and pathogen-rich fungal lineages. Studies in Mycology 62, 111-119.

Hall TA. 1999 - BioEdit: a user-friendly biologicalsequence alignment editor and analysis program for Windows 95/98/NT. Nucleic Acids Symposium Series 41, 95-98

Hongsanan S, Li YM, Liu JK, Hofmann T et al. 2014 - Revision of genera in Asterinales. Fungal Diversity 68, 1-68.

Hongsanan S, Sánchez-Ramírez S, Crous PW, Ariyawansa HA et al. 2016 - The evolution of fungal epiphytes. Mycosphere 7, 1690-1712.

Hyde KD, Jones EBG, Liu JK, Ariyawansa HA et al. 2013 - Families of Dothideomycetes. Fungal Diversity 63, 1-313.

Jayasiri SC, Hyde KD, Ariyawansa HA, Bhat J et al. 2015 - The Faces of Fungi database: fungal names linked with morphology, phylogeny and human impacts. Fungal Diversity 74, 3-18.

Katoh K, Standley DM. 2013 - MAFFT multiple sequence alignment software versions 7: improvements in performance and usability. Molecular Biology and Evolution 30, 772780.

Labandiera CC, Sepkoski JJ Jr. 1993 - Insect diversity in the fossil record. Science 261, 310-315.

Liu YJ, Whelen S., Hall BD. 1999 - Phylogenetic relationships among ascomycetes: evidence from an RNA polymerase II subunit. Molecular Biology and Evolution 16, 1799-1808.

Lumbsch HT, Huhndorf SM. 2010 - Outline of Ascomycota - 2009. Fieldiana Life and Earth Sciences 1, 1-60.

Maharachchikumbura SSN, Hyde KD, Jones EBG, McKenzie EHC et al. 2016 - Families of Sordariomycetes. Fungal Divers 79, 1-317.

Mapook A, Boonmee S, Ariyawansa HA, Tibpromma S et al. 2016a - Taxonomic and phylogenetic placement of Nodulosphaeria. Mycological Progress 15, Article 34.

Mapook A, Hyde KD, Dai DQ, Li JF et al. 2016b - Muyocopronales, ord. nov., (Dothideomycetes, Ascomycota) and a reappraisal of Muyocopron species from northern Thailand. Phytotaxa 265, 225-237.

Minter DW, Peredo HL, Watson AT. 2007 - Acrospermum chilense sp. nov. from Chile and the Acrospermales ord. nov. Bulletin of the Botanical Society of Argentina 42, 107-112.

Müller E, Arx JA von. 1962 - Die Gattungen der didymosporen Pyrenomyceten. Beiträge zur Kryptogamenflora der Schweiz 11, 1-922.

Nylander JAA. 2004 - MrModeltest 2.0. Program distributed by the author. Evolutionary Biology Centre, Uppsala University

Page RDM. 1996 - TreeView: an application to display phylogenetic trees on personal computers. Comput Appl Biosci 12, 357-358.

Pang KL, Hyde KD, Alias SA, Suetrong S, Jones EBG. 2013 - Dyfrolomycetaceae, a new family in the Dothideomycetes, Ascomycota. Cryptogamie, Mycologie 34, 223-232.

Pérez-Ortega S, Garrido-Benavent I, Grube M, Olmo R, de los Ríos A. 2016 - Hidden diversity of marine borderline lichens and a new order of fungi: Collemopsidiales (Dothideomyceta). Fungal Diversity 80, 285-300.

Prieto M, Baloch E, Tehler A, Wedin M. 2013 - Mazaedium evolution in the Ascomycota (Fungi) and the classification of mazaediate groups of formerly unclear relationship. Cladistics 29 , 296-308. 
Rambaut A, Suchard MA, Drummond AJ. 2014 - Tracer v 1.6, available from: http://tree.bio.ed.ac.uk/software/tracer/Rehner SA, Samuels GJ (1994) Taxonomy and phylogeny of Gliocladium analysed from nuclear large subunit ribosomal DNA sequences. Mycological Research 98, 625-634.

Rambaut A. 2009 - FigTree v1.4: Tree figure drawing tool. Available: http://tree.bio.ed.ac.uk/software/figtree/.

Retallack GJ. 1995 - Permian-triassic life crisis on land. Science 267, 77-80.

Riddle LW. 1920 - Observations on the genus Acrospermum. Mycologia 12, 175-181.

Rikkinen J. 2003 - Calicioid lichens from European Tertiary amber. Mycologia 95, 1032-1036.

Ryder G, Fastovsky DE, Gartner S. 1996 - The Cretaceous-Tertiary event and other catastrophes in earth history. Geological Society of America 307, 1-550.

Schoch CL, Wang Z, Townsend JP, Spatafora JW. 2009 - Geoglossomycetes cl. nov., Geoglossales ord. nov. and taxa above class rank in the Ascomycota tree of life. Persoonia 22, 129-138.

Shen SZ, Crowley JL, Wang Y, Bowring SA et al. 2011 - Calibrating the end-Permian mass extinction. Science 334, 1367-1372.

Sydow H, Sydow P. 1914 - Fungi from northern Palawan. Philippine Journal of Science Section C Botany 9, 157-189.

Vijaykrishna D, Jeewon R, Hyde KD. 2006 - Molecular taxonomy, origins and evolution of freshwater ascomycetes. Fungal Diversity 23, 351-390.

Vilgalys R, Hester M. 1990 - Rapid genetic identification and mapping of enzymatically amplified ribosomal DNA from several Cryptococcus species. Journal of Bacteriology 172, 4239 4246.

Visscher H, Brinkhuis H, Dilcher DL, Elsik WC et al. 1996 - The terminal Paleozoic fungal event: evidence of terrestrial ecosystem destabilization and collapse. Proceedings of the National Academy of Sciences of the United States of America 93, 2155-2158.

White TJ, Bruns T, Lee S, Taylor J. 1990 - Amplification and direct sequencing of fungal ribosomal RNA genes for phylogenetics. In: Innis MA, Gelfand DH, Sninsky JJ, White TJ (eds) PCR protocols: a guide to methods and applications, Academic, San Diego, 315-322.

Wijayawardene NN, Crous PW, Kirk PM, Hawksworth DL et al. 2014 - Naming and outline of Dothideomycetes 2014. Fungal Diversity 69, 1-55.

Wu HX, Schoch CL, Boonmee S, Bahkali AH et al. 2011 - A reappraisal of Microthyriaceae. Fungal Diversity 51, 189-248. 